\section{GOLD STANDARD \\ ANTI-MICROBIAL}

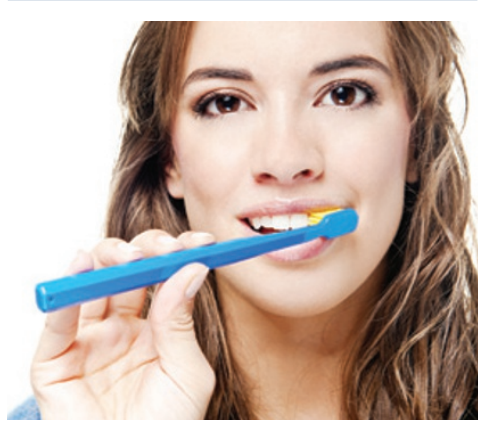

Oral healthcare experts Curaprox will reveal their new branding at this year's Showcase. Delegates at the Curaprox stand (N07) will also experience live demos following the 'touch 2 teach' philosophy from Curaprox's iTOP (individually Trained Oral Prophylaxis). Visitors will also discover Curasept, non-staining $\mathrm{CHX}$ toothpaste and gel formulations - products that contain the industry's gold standard anti-microbial without its side effects.

The Curaprox team will also be at hand to share the new generation of interdental brushes - the CPS Prime range. Made from nickel free CURAL surgical wire, these interdental brushes offer extreme durability with the thinnest of cores - so they last up to five times longer than other brushes on the market.

To learn more make sure you visit stand, N07.

For more information call 01480862084.

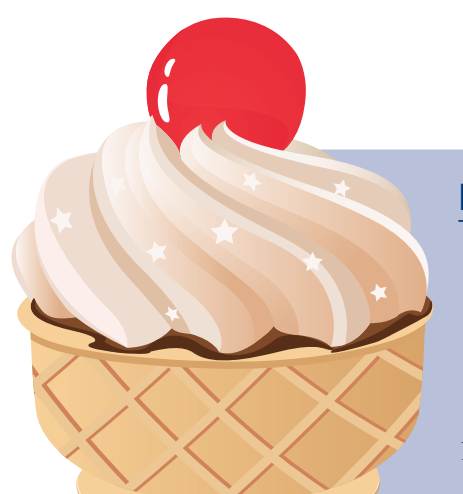

\section{ICE CREAM AND PATIENT PLAN ADVICE}

According to recent consumer research, Denplan is the third most widely recognised healthcare brand in the UK, behind only BUPA and AXA PPP, with an astonishing 50\% recognition rate (Simplyhealth/HPI based on 1,178 responses).

As the only dental payment plan specialist that patients can ask for by name, Denplan uses its consumer brand identity to help you grow your business. Go along to stand F10 to enjoy an ice cream and find out how.

That's not all - Denplan will also be announcing some
Sensonic Professional Plus Sonic Toothbrush has up to $25 \%$ faster bristle speed for $29 \%$ better plaque removal than other sonic toothbrushes available. The combination of the two was proven $70 \%$ more effective than a leading sonic toothbrush for reducing gingival bleeding.

To find out more, don't forget to visit the experts on stand H06 at BDTA Dental Showcase 2013. Or visit the website at www. waterpik.co.uk.

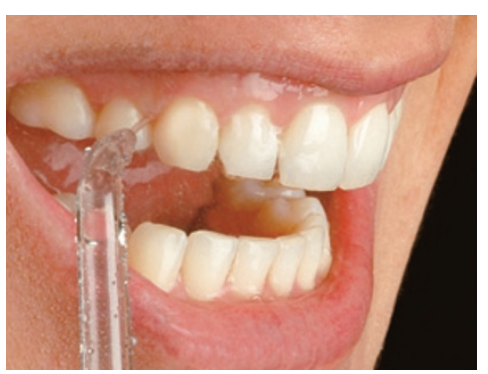

exciting new product enhancements at this year's BDTA Dental Showcase to benefit both practices and patients, so go along and talk to one of the friendly team at Denplan's stand to find out more.

For more information about Denplan, visit the website at www.denplan.co.uk or telephone 08001699962. 